\title{
Method for CFD Simulation of Propellant Slosh in a Spherical Tank
}

\author{
David J. Benson ${ }^{1}$ and Paul A. Mason ${ }^{2}$ \\ NASA Goddard Space Flight Center, Greenbelt, MD, 20771
}

\begin{abstract}
Propellant sloshing can impart unwanted disturbances to spacecraft, especially if the spacecraft controller is driving the system at the slosh frequency. This paper describes the work performed by the authors in simulating propellant slosh in a spherical tank using computational fluid dynamics (CFD). ANSYS-CFX is the CFD package used to perform the analysis. A $\mathbf{4 2}$ in spherical tank is studied with various fill fractions. Results are provided for the forces on the walls and the frequency of the slosh. Snapshots of slosh animation give a qualitative understanding of the propellant slosh. The results show that maximum slosh forces occur at a tank fill fraction of 0.4 and 0.6 due to the amount of mass participating in the slosh and the room available for sloshing to occur. The slosh frequency increases as the tank fill fraction increases.
\end{abstract}

\section{Introduction}

CLOSH behavior is observed when a bucket of water is carried from one location to another. As the person $\checkmark$ carrying the bucket walks, the back and forth the motion of the person's hand motion causes the water to move back and forth in the bucket (slosh). When the bucket is set down, the water continues to slosh back and forth within the bucket and the forces exerted on the bucket walls cause the bucket to rock back and forth or shake.

This same phenomenon occurs with a spacecraft's propellant when maneuvers are performed in space.

During a spacecraft maneuver propellant slosh can be excited by the spacecraft control system. The control system then uses thrusters to control the slosh resulting in the use of propellant. In some cases control maneuvers can excite the slosh further. In extreme cases, slosh can result in mission failure. For spinning spacecraft a nutation can be introduced by sloshing propellant. Problems with slosh have occurred on ATS-V, Intelsat IV series spacecraft, NEAR Shoemaker mission, and Gravity Probe B. ${ }^{1}$ As the ratio between propellant and dry mass increases, the impact of slosh also increases, causing a significant effect on attitude control, performance, and stability. For missions requiring large amounts of propellant for orbit insertion, orbit maintenance, or momentum unload, it is imperative that slosh dynamics are well understood.

Slosh dynamics in a low gravity environment are very nonlinear and cannot easily be modeled. Computational fluid dynamics (CFD) can be used to provide an accurate description of the fluid dynamics. ${ }^{2,3}$ Due to CFD being computationally expensive, other computationally inexpensive modeling techniques can be used to provide a first order description of the slosh dynamics. The most common first order model is an equivalent mechanical model such as a pendulum model. ${ }^{4}$ Equivalent mechanical models can be used to design controllers and determine stability margins. Because CFD results are used to create these simpler models, it is important that the CFD results accurately describe the resulting forces and torques on the propellant tank wall. In addition, accurate CFD results will also provide better estimates of the modal frequency and viscous damping of the sloshing. Because of the cost of writing a CFD code it is often more cost effective to use proven commercially available codes such as ANSYS-CFX.

In the following sections, a method for simulating sloshing in a spherical tank with a diameter of 42 inches, using ANSYS-CFX, in a constant accelerating environment, and with a constant rate, is presented. For completeness, various tank fill fractions are simulated. The results provided are not compared to experimental data, but are reviewed by professionals who are familiar with the forces applied to a spacecraft to verify that the results are reasonable and realistic.

\footnotetext{
${ }^{1}$ Student Trainee, Propulsion Branch, 597, Graduate Student, Purdue University, and AIAA Student Member

${ }^{2}$ Aerospace Engineer, Attitude Control Systems, 591, and AIAA member 


\section{Experimental Setup}

This analysis models a spherical propellant tank with a diameter of 42 in with propellant fill fractions of $0.2,0.4$, 0.6 , and 0.8. No propellant management devices are included (bare tank). Oxidizer and hydrazine are the propellants modeled (see Table 1). The viscosity shown in Table 1 is multiplied by 1.6 due to CFD simulations' tendency to inaccurately model viscosity in real fluid. This number was chosen after discussion with professionals familiar with CFD simulations. An acceleration of $-0.24 \mathrm{~m} / \mathrm{s}^{2}$ along the $\mathrm{z}-$ axis is imposed with the initial position of the propellant at a 45 deg angle from the negative $z$-axis in the $y-z$ plane (See Fig. 1). An acceleration of $0.24 \mathrm{~m} / \mathrm{s}$ is on the high end of the accelerations seen on various missions. The 45 degrees angle is the worst case initial propellant angle with respect to $\mathrm{CM}$ motion. This is a conservative estimate since the slosh spill over angle for a spherical tank is approximately 30 degrees.

ANSYS-CFX is the commercial CFD program used to obtain the results in this paper. ANSYS-CFX uses a control volume method. This means that the flow domain is discretized using a mesh and the governing equations are integrated over the control volume. ${ }^{7}$ The cases in this paper consist of an initializing simulation (static simulation) and a transient simulation.

To initialize the fluid at an angle of $45 \mathrm{deg}$ from the negative $z$-axis, a steady-state simulation is run in ANSYS-CFX. The fluid is oriented at the desired position by causing the acceleration vector to point at a $45 \mathrm{deg}$ angle from the $z$-axis in the $y-z$ plane. Because this only initializes the fluid position, the exact acceleration chosen is not important as long as the acceleration is not too high. If the acceleration is too high it will cause the first few data points in the transient simulation to be incorrect while the solver tries to get the fluid to the correct pressure. Air is modeled as the pressurizing gas with a tank pressure of $0 \mathrm{psi}$, though helium would be used in an acutal tank with an actual pressure of 250 psi. Because the propellants are much denser and more viscous than the gas, and because the simulation treats all the fluids as

Table 1. Propellant properties ${ }^{5,6}$.

\begin{tabular}{|c|l|l|}
\hline Property & $\begin{array}{c}\text { Hydrazine } \\
\left(\mathbf{N}_{2} \mathbf{H}_{4}\right)\end{array}$ & $\begin{array}{c}\text { Oxidizer } \\
\left(\mathbf{N}_{2} \mathbf{O}_{4}\right)\end{array}$ \\
\hline $\begin{array}{c}\text { Molar Mass } \\
(\mathbf{k g} / \mathbf{k m o l})\end{array}$ & 50.06 & 92.011 \\
\hline $\begin{array}{c}\text { Density } \\
\left(\mathbf{g} / \mathbf{c m}^{\mathbf{3}}\right)\end{array}$ & 1.032 & 1.443 \\
\hline $\begin{array}{c}\text { Dynamic Viscosity } \\
(\mathbf{c P})\end{array}$ & 1.44464 & 0.88 \\
\hline $\begin{array}{c}\text { Surface Tension } \\
(\mathbf{d y n e} / \mathbf{c m})\end{array}$ & 66.5 & 26.5 \\
\hline
\end{tabular}

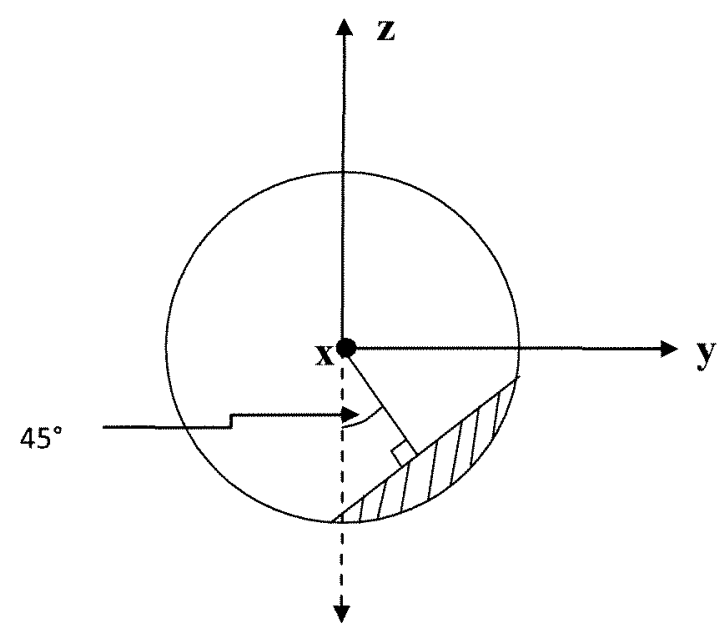

Figure 1. Coordinate system of spherical tank with the initial position of the propellant shown. incompressible, the results will not change significantly if the tank is pressurized and if helium instead of air is used as the pressurizing gas. The buoyancy reference density is set to the density of air because it is the least dense of the two fluids in the tank. Setting the reference density this way allows the air to be nearly the same pressure everywhere and gives the liquid a hydrostatic pressure. ${ }^{8}$ The homogeneous model is used due to software license restrictions. One study has shown that CFD turbulence models are recommended for slosh simulations. ${ }^{2}$ However, another study that compared turblulent models and laminar flow models has shown that the laminar flow models are more conservative, and therefore also acceptable. ${ }^{3}$ Laminar flow is assumed for this simulation to be more consevative. Surface tension forces are included. For better accuracy a high-resolution advection scheme is used.

The forces on the tank due to slosh are obtained from the transient simulations. The transient runs have uniform time steps of $0.02 \mathrm{sec}$ with total slosh time simulated varying from $50 \mathrm{sec}$ to $100 \mathrm{sec}$. The time step size is chosen because it captures the fluid physics while keeping the run time of the simulation reasonable. The time simulated allows for the slosh to dampen out until only the dominant frequency motion remains. The settings used for the static simulation described in the previous paragraph are repeated for the transient simulation except that the acceleration vector is pointed in the negative $z$-direction. The forces in the $x-, y-$, and $z$-direction are outputted by the ANSYS-CFX Solver. The frequency of the force oscillation is found using a Discreet Fourier transform. The 
results are further processed to create an animation of the fluid slosh by looking at the volume fraction in the $y-z$ plane (main slosh plane) as a function of time. This animation provides a visual check to make sure the slosh seems physically likely as well as gives insight into propellant slosh behavior.

\section{Results and Discussion}

\section{A. Hydrazine Slosh Forces}

Figure 2 shows the results for the forces on the walls broken into $\mathrm{x}, \mathrm{y}$, and $\mathrm{z}$ components with the hydrazine as the propellant. The numbers shown in the legend are the fill fractions at which the simulations are run.

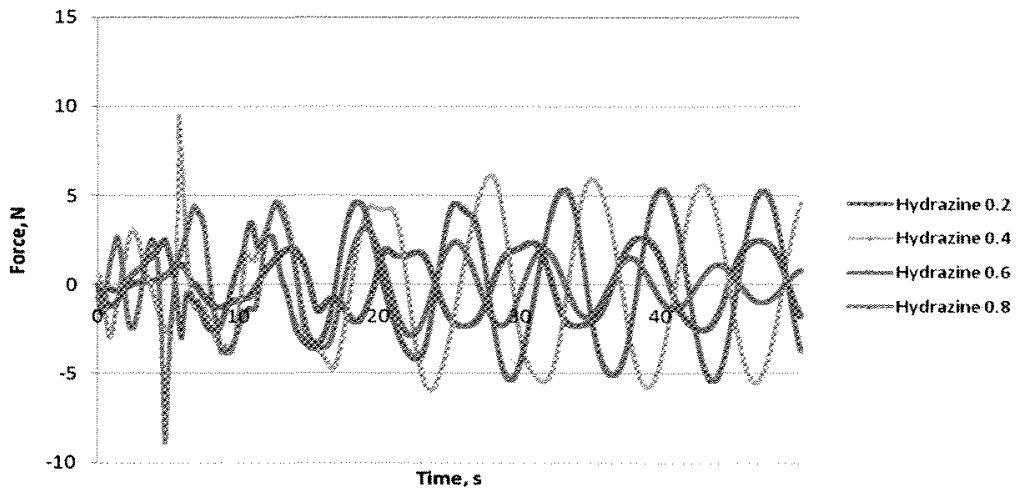

a)

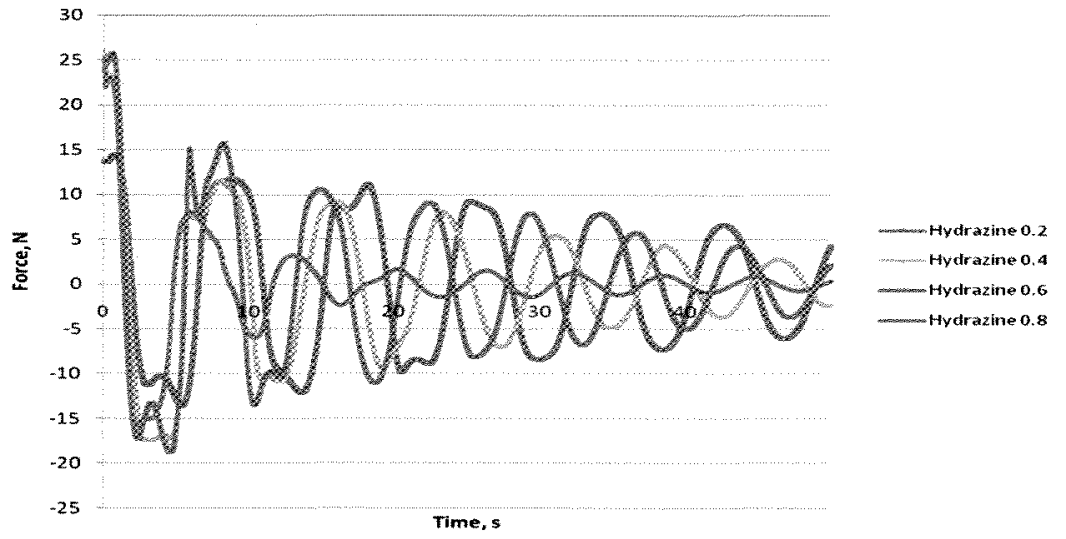

b)

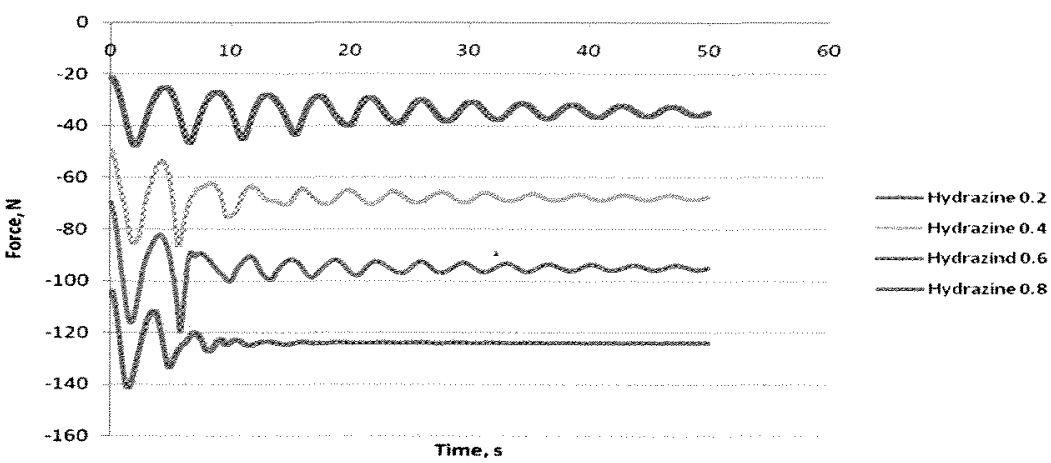

c)

Figure 2. Force vs time plot for hyrdazine in a) $\mathrm{x}$-direction, b) y-direction, and c) $\mathrm{z}$-direction. 
Fill fractions of 0.4 and 0.6 create the largest forces. This can be explained by a mechanical equivalent model of the slosh. Dodge pointed out that propellant slosh can be modelled by using a mechanical model such as a pendulum. ${ }^{4}$ The mass of the pendulum in this approximation is a fraction of the total mass of the propellant. The difference between the pendulum mass and propellant mass is added to the model as a stationary mass (no sloshing behavior). ${ }^{4}$ Fill fractions of 0.4 and 0.6 have more space to slosh thus allowing a larger percentage of the propellant mass to slosh. This means a larger percentage of the mass is considered as a "swinging" pendulum mass in the equivalent mechanical model. Conversely, at a fill fraction of 0.8 there is less room for sloshing and a smaller percentage of the propellant mass is considered as a "swinging" pendulum mass.

Slosh forces are three dimensional in a spherical tank even though the force is applied in one plane. Forces in the $\mathrm{x}$-direction (Fig. 2a) are the smallest because these forces are caused by the swirling motion of the propellant. The breaking up and recombining of the fluid at the beginning of the simulation causes the unpredictability (nonlinear dynamics) shown at the beginning of Fig 2a. These nonlinearities cannot be modeled by equivalent mechanical models.

Forces in the $y$-direction are larger than forces in the $x$-direciton because the acceleration of the propellant is occuring in the $y-z$ plane. Fig. $2 b$ also shows some unpredictability at the beginning of the simulation though more muted due to the resolution of the plot compared to Fig. 2a. As the simulation continues, the sloshing of the fluid settles into a dominant frequency and slowly dampens out due to viscous forces in the fluid. The damping is most apparent in Fig. $2 \mathrm{~b}$ at a fill fraction of 0.8 . The tank is close to full so the initial movement of the propellant causes large forces but the forces dampen out quickly due to the large amount of propellant present to viscously dampen out the forces.

The offsets of the forces in the z-direction in Fig. 2c represent the stationry mass in the pendulum example and the magnitude of the oscillations represents the mass that is sloshing, e.g. the "swinging" pendulum mass.

\section{B. Oxidizer Slosh Forces}

The oxidizer results are shown in Figure 3. Similar trends to the slosh forces caused by the hydrazine are shown in the oxidizer plots. However, the oxidizer exhibits higher amplitude forces than the hydrazine. This is as expected because the oxidizer is denser than hydrazine. The trends followed include a damping of forces over time, many frequencies in the force oscillaiton at the beginning of the simulation, and offsets of the force in the z-direction.

It is intersting to note that at a fill froaction of 0.8 the slosh in the $\mathrm{x}$-direction is minimal. It is unkown why this happens with oxidizer and not with hydrazine. Further study in this area would be useful for future spacecraft design.

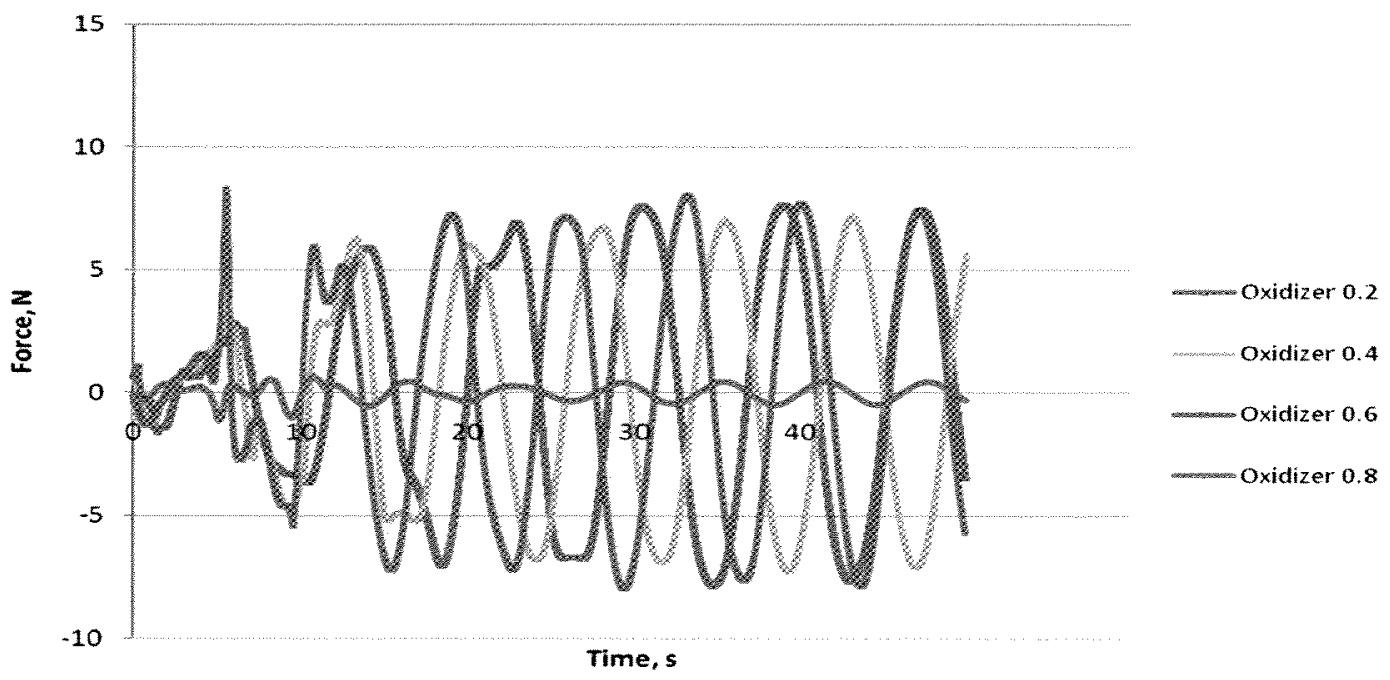

a) 


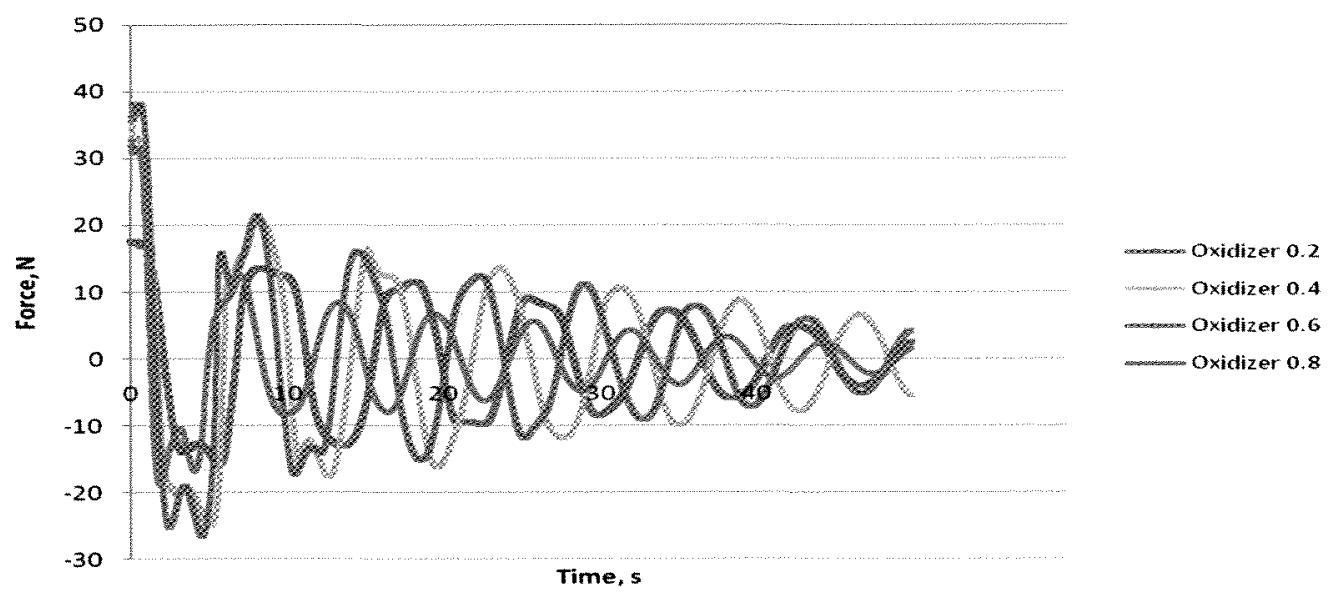

b)

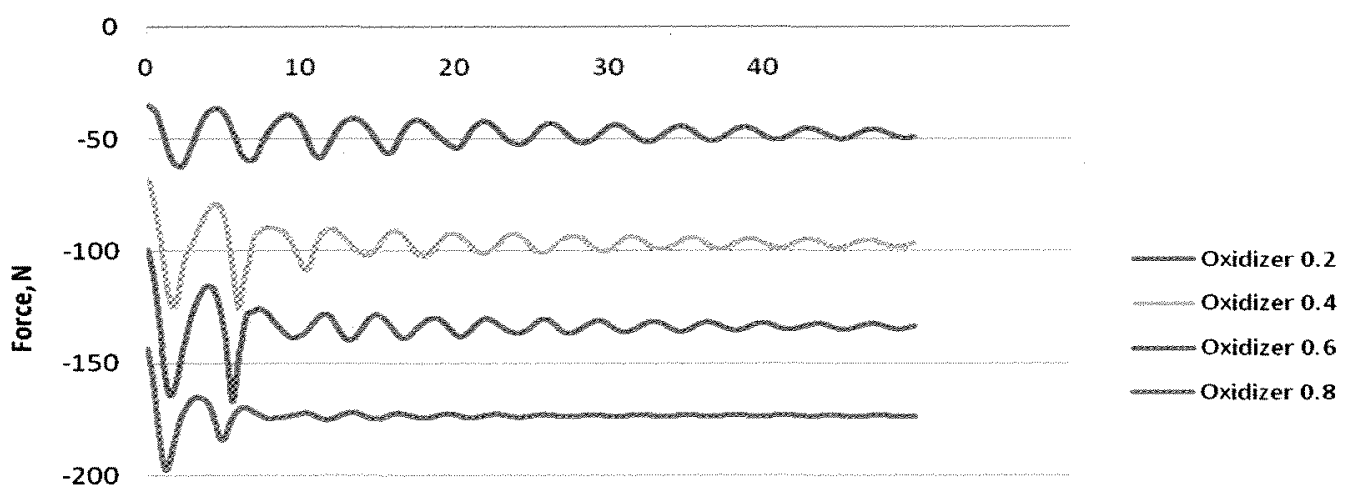

$-250$

Time, s

c)

Figure 3. Force vs time plot for oxidizer in a) $\mathrm{x}$-direction, b) $\mathrm{y}$-direction, and c) $\mathrm{z}$-direction.

\section{Slosh Frequency Results}

Table 2 provides the dominant frequencies for the forces exerted on tank walls by the sloshing propellant. The data indicates that the dominant frequency for a spherical tank is a function of fill fraction and not of propellant used, except for a fill fraction of 0.8 . This trend is followed except in the z-direction at a fill fraction of 0.8 . This difference may be related to the lack of sloshing in the $\mathrm{x}$-direction seen in Fig. $3 \mathrm{a}$ and/or it might suggest that mass is important at higher fill fractions. Further studies may be able to help us understand this phenomenon better. These findings could have significant implications for developing a pendulum model equivalent of the slosh independent of tank fluid since initial results seem to indicate that Table 2. Dominant frequency results using discrete Fourier transform.

\begin{tabular}{|c|c|c|c|c|c|c|}
\hline & \multicolumn{3}{|c|}{ Hydrazine Frequency $(\mathrm{Hz})$} & \multicolumn{3}{c|}{ Oxidizer Frequency $(\mathrm{Hz})$} \\
\hline Fill Fraction & $\mathrm{x}$ & $\mathrm{y}$ & $\mathrm{z}$ & $\mathrm{x}$ & $\mathrm{y}$ & $\mathrm{z}$ \\
\hline $\mathbf{0 . 2}$ & 0.1221 & 0.1221 & 0.2319 & 0.1221 & 0.1221 & 0.2319 \\
\hline $\mathbf{0 . 4}$ & 0.1343 & 0.1343 & 0.2563 & 0.1343 & 0.1343 & 0.2563 \\
\hline $\mathbf{0 . 6}$ & 0.1343 & 0.1343 & 0.2685 & 0.1343 & 0.1343 & 0.2685 \\
\hline $\mathbf{0 . 8}$ & 0.1587 & 0.1587 & 0.3174 & 0.1587 & 0.1587 & 0.3052 \\
\hline
\end{tabular}

frequency is a function of the type of fluid used at lower fill fractions. 


\section{Slosh Visualization}

By outputting the propellant volume fraction as an animation, some important qualitative checks can be made. Further, by comparing the animation with the force and frequency results some important conclusions can be drawn about sloshing. Figure 4 shows hydrazine at a fill fraction of 0.4 throughout the simulation. Fig. 4a shows the fluid starting at $45^{\circ}$ from the vertical position. When the simulated spacecraft acceleration occurs in the positive $\mathrm{z}$ direction the propellant feels an acceleration in the negative $z$-direction. This causes the sloshing motion shown in Fig. 4b through Fig. 4f. The severity of the slosh causes the propellant to break apart as it sloshes back and forth in

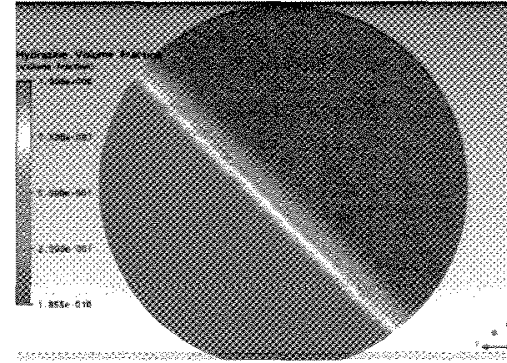

a)

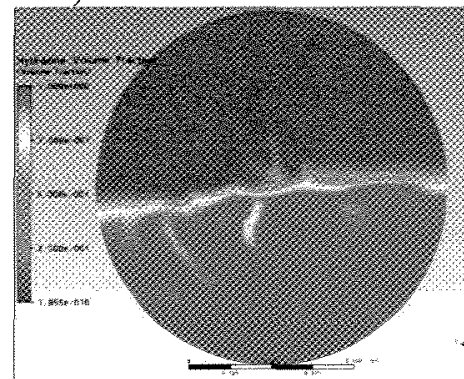

d)

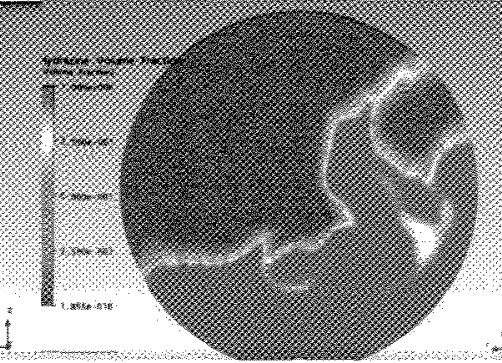

b)

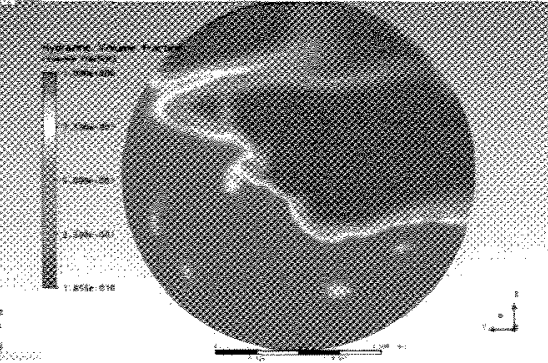

c)

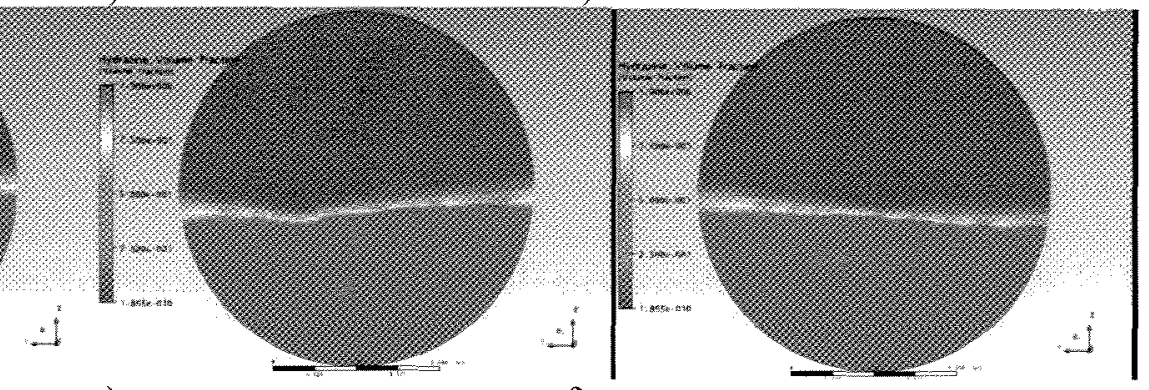

f)

Figure 4. Snapshots from animation output of volume fraction for Hydrazine at 0.4 fill fraction at approxiamately a) $0 \mathrm{sec}$, b) $3 \mathrm{sec}$, c) $5 \mathrm{sec}$, d) $23 \mathrm{~s}$, e) $35 \mathrm{sec}$, and f) $48 \mathrm{sec}$.

Fig. $4 \mathrm{~b}$ and Fig. $4 \mathrm{c}$. At the beginning of the simulation there are many frequencies present due to the chaotic nature of the sloshing motion of the propellant as can be seen in Fig. 4b through Fig $4 \mathrm{~d}$. These nondominant frequencies quickly die out and the dominant frequency is all that is left as shown in Fig. 4e and Fig. 4f. This dominant frequency is dampened out more gradually because it is lower than the other frequencies. It is important to note that many spacecraft maneuvers will not last long enough for all of the non-dominant frequencies to dampen out.

\section{Conclusion}

Propellant slosh in spacecraft can cause unwanted disturbances, which can be excited by the spacecraft control system if the slosh is not understood. Due to the complexity of propellant slosh in a tank and the difficulty of getting experimental data for slosh in a low-g environment, computational fluid dynamics (CFD) can be used as an effective design/analysis tool. The paper presented gives a method for using ANSYS-CFX to find the forces and the frequency of the forces on a spherical tank. The results presented show that CFD is able to capture the complex motion of the slosh, output forces on the propellant tank, and through a Discreet Fourier Tranform give the dominant frequency of the slosh. The force results show that maxium forces occur at fill fractions of 0.4 and 0.6 . The results also seem indicate that the frequency of the slosh is a function of fill fraction for this geometry and not a function of propellant used. This becomes important if mechanical models are to be used to approximate the slosh. Further work could include running simulations with varying grids to see the effect of the grid on results, further analysis of lack of swirling motion for a fill fraction of 0.8 , trying different tank sizes and propellant types to see if the frequency trends still hold, including propellant management devices as part of the simulation, and comparison with physical experiment data. 


\section{Acknowledgments}

The authors would like to thank Alison Rao of NASA Goddard Space Flight Center's propulsion branch and Henry Fitzpatrick of the attitude controls branch for the help in applying the CFD software to the problem presented in this paper as well as help with creating appropriate CFD grids. We would also like to thank Maria Lang, Valerio Moro, Kevin Bonanne, and Jason Lynch as members of the NASA Goddard Space Flight Center Summer Intern Cheetah Group for their input in checking our work and suggesting improvements to the simulation. Finally, we would like to thank NASA Goddard Space Flight Center for providing the facilities and software to perform the research.

\section{References}

'Vreeburg, J.P.B., "Spacecraft Maneuvers and Slosh Control," IEEE control System Magazine, Vol. 25, No. 3, June, 2005, pp. 12 - 16.

${ }^{2}$ Godderidge, B., Tan, M., Turnock, S., \& Earl, C., "A Verification and Validation Study of the Application of Computational Fluid Dynamics to the Modeling of Lateral Sloshing," Fluid Structure Interaction Research Group, Ship Science Report No 140, Southampton University, Southampton, UK, Aug. 2006

${ }^{3}$ Marsell, B., Gangadharan, S., Chatman, Y., Sudermann, J., Schlee, K., \& Ristow, J., "A CFD Approach to Modeling Spacecraft Fuel Slosh," 47th AIAA Aerospace Sciences Meeting Including The New Horizons Forum and Aerospace Exposition, Disc 1, AIAA, 2009.

${ }^{4}$ Dodge, F. T "The New "Dynamic Behavior of Liquids in Moving Containers", Southwest Research Institute, Technical Report, San Antonio, TX, 2000.

${ }^{5}$ Schmidt, E.W., Hydrazine and Its Derivatives: Preparation, Properties, Applications, John Wiley \& Sons Inc., New York, 1983.

${ }^{6}$ Addison C.C., "Dinitrogen Tetroxide, Nitric Acid, and Their Mixtures as Media for Inorganic Reactions," Chemical Review, Vol. 80, No. 1, 1980, pp. 21-39.

${ }^{7}$ ANSYS, ANSYS CFX-Solver Theory Guide, ANSYS Inc., Canonsburg, PA, 2009

${ }^{8}$ ANSYS, ANSYS CFX-Solver Modeling Guide, ANSYS Inc., Canonsburg, PA, 2009 